\title{
Teaching Social Informatics as a knowledge project
}

\author{
Iver Jackewitz, Michael Janneck, Detlev Krause, Bernd Pape and Monique \\ Strauss \\ Department for Informatics, University of Hamburg, Vogt-Kölln-Straße 30, D-22527 Hamburg, \\ Germany
}

\{jackewitz|janneck|krause|pape|strauss\}@informatik.uni-hamburg.de

Key words: Collaborative Learning, Curriculum Policies, Didactics of Informatics, Higher Education

\begin{abstract}
Teaching Social Informatics poses the challenge of portraying future scenarios of technology development and use as well as criticising them. We envision meeting this challenge by offering students an authentic, interdisciplinary, educational framework, providing real-life, hands-on experiences. In this paper, we will first develop the idea of "knowledge projects" as a means to encourage such educational settings. Then we will focus on our efforts to develop a Web-based software system, named CommSy, designed to support communication and co-ordination in learning communities involved in knowledge projects.
\end{abstract}

\section{INTRODUCTION}

The term "Social Informatics", coined by Kling (1999) was defined as the interdisciplinary study of the design, uses and consequences of information technologies that takes into account their interaction with institutional and cultural contexts. Enabling students to better understand the interaction of information technologies and their fields of application is a central issue in Social Informatics curricula, because, in this subject area, we train future specialists who will play an active role in developing new information technology solutions for people in other fields. Teaching Social Informatics

The original version of this chapter was revised: The copyright line was incorrect. This has been corrected. The Erratum to this chapter is available at DOI: 10.1007/978-0-387-35663-1_34 
has to deal with the ambiguity of promising future scenarios of technology development and use on the one hand and with harsh critics of these scenarios on the other.

One promising scenario, for example, is the $4 \mathrm{~A}$ scenario:

"Any one can get any document anytime and anywhere. There are instantiations of $4 \mathrm{~A}$ such as providing any researcher with all of the documentary materials that they want for their research, even if they are traveling for a month; or providing any doctor with a complete medical record for any patient, anytime, anywhere." (Kling and Star 1998)

Living and working in the 4A scenario would require various professional, social and technical skills in dealing with unfamiliar problems and becoming acquainted with ever changing circumstances. This comprises, for example, the ability to get to know colleagues, grasp their perspectives on current problems, and to take up work done by them, all in a short period of time. Therefore, it also seems essential that we should make effective and efficient use of software tools that support such project teams. However, scenarios like $4 \mathrm{~A}$ are criticized because they too easily trivialise the interaction of design, uses and consequences of information technologies with their context:

"by homogenizing people and places into 'everyman' and 'everywhere'. The various roles that people play in work groups are ignored and stereotyped. The ways that organizations structure information is also treated only as a barrier, unless materials are accessible $4 \mathrm{~A}$. The different kinds of resources (and skill sets) of organizations and groups are also all homogenized in 4A scenarios." (Kling and Star 1998)

To offer our students the opportunity to learn the necessary professional, social and technical skills to live and work in a probable 4A scenario, and to take a critical stance on the prospective development at the same time, we consider it is not sufficient to merely present this ambiguity. In our opinion, students should have the opportunity to gain experience in technology use, in new forms of work organization and in related ways of organizing their lives during their studies. To offer our students authentic educational settings, we regard studies in Social Informatics as a "knowledge project". In this paper, we will first describe what we mean by "studying Social Informatics as a knowledge project". We examine two views on knowledge projects that guided us in our efforts to develop software support for teaching Social Informatics and then focus on the resulting software system CommSy. 


\section{KNOWLEDGE PROJECTS}

What is a "knowledge project"? Let us start defining the term by explaining its constituent parts. We consider "knowledge" to be a contextdependent representation of information (Schultze 1998) and "projects" we define as learning opportunities that emphasize practical, problem-centered, activity-oriented learning (Gudjons 1997). Therefore, when we speak of "knowledge projects", we are basically talking about context-based knowledge obtained in hands-on, real-world settings, facing real problems which are of actual importance to students and to other people.

We identify four major aspects of knowledge projects:

Acquiring knowledge. As mentioned above, we do not consider knowledge as context-independent. However it is not completely individualized either. Knowledge may be established by communities and is carried on through traditions and customs. Imparting knowledge is thus not an activity that is passively received by one or more learners. Acquiring knowledge has to be seen as an active endeavour integrating "new" pieces of information into existing structures of knowledge and experience in which social and group experiences play a major role. From this viewpoint, faculty staff are seen as facilitators (Rogers 69) who try to spark off and encourage individual and group learning processes - rather than "classical" lecturers who strive for a close match of student know-how with their own.

Multiple players and multiple perspectives. Learning and acquiring knowledge in a university context always involves multiple players especially students, teachers and professionals from the respective vocational field - with different views and experiences. These multiple perspectives should not be ignored or streamlined. They should be taken into account and used to enrich the learning experiences of those involved. Thus, studying informatics as a knowledge project means that different standpoints and experiences are emphasized, dealt with explicitly and tied together into a broader perspective. This implies on the one hand that the students' viewpoints and experiences are taken as seriously as those of "experts", but on the other hand it means that different persons never learn - or should be forced to learn - exactly the same things. Individual experiences serve as a starting point, an anchor from which further learning experiences are developed in the respective context and can be shared with others.

Crossing boundaries. While it is important to have multiple perspectives within one community of practice, we also emphasize the importance of multidisciplinary exchange and communication. In modern vocational fields professional barriers have become permeable. A lot of tasks require a broad range of different skills from different professional backgrounds. We emphasize the importance of gaining different vocational competencies not 
only in professional life, but also during university education.

IT support. IT support in university education should "support" those involved in a way that actually makes work more efficient and effective or allows for new ways of communication, cooperation or storage of information. Another important aspect of IT support in university education lies in the opportunity for students to gain competencies in the use and handling of "new" media such as the Internet.

The concept of university education as a knowledge project implies two perspectives. Firstly, it can serve as a model for organizing singular learning activities (lectures, study groups). This emphasis on project-based learning lets students work on complex problems in small teams over an extended period of time (Pape et al 2002). Secondly, we pursue the goal of understanding and organizing university courses of study - in our case particularly Social Informatics - as a whole as knowledge projects. This means we have to bridge the gaps between separate courses and strengthen connections between different parts and contents of university study courses. The keywords of continuity, transparency and coherency regarding different content and methodology in university education are vital in this respect.

\section{SOFTWARE SUPPORT FOR KNOWLEDGE PROJECTS}

In our research and software development process, we strive to provide software support for knowledge projects. Since there is always a set of different traditional and "new" media used in educational settings, we don't try to replace them with a system which claims to cover all communication needs in university teaching. We consider this neither feasible nor desirable. In acquiring competences in the use of technical support for communication and coordination, it is more important to strive to develop the capability of handling a variety of technologies. Knowing which medium is appropriate to which communicative need and knowing how to express this need in the selected medium are necessary skills for living and working in a networked world. That is why we concentrate on the two perspectives on knowledge projects as learning environments in our research and development.

By concentrating on knowledge projects, we emphasize the situated nature of learning processes. Learning processes do not happen anytime but at a particular time, consisting not just of anyone but of particular persons, motivated not by any reason but by particular reasons and not working with any documents but with particular ones. Our software development aims to support faculty and students to take part in knowledge projects in an active and reflective manner. 


\section{COMMSY}

CommSy (CommSy 2001) stands for Community System and is a webbased system to support communication and coordination in learning communities. In its latest version CommSy comprises three components: CommSy Project Rooms support close cooperation in closed work groups with 10 - 30 members. CommSy Archive provides a collection of study materials and student work which is partly accessible worldwide. CommSy Campus links the archive and the project rooms, offers information on courses and extracurricular activities and thus provides central access to the system.

CommSy Project Rooms are designed to support singular learning activities as knowledge projects (Pape et al 2002). By virtue of its functional scope, the system supports central activities of a learning project as outlined by Gudjons. It supports communication in multiple ways: News and events can be announced, specific topics can be discussed in discussion forums, each member has a personal homepage to present him/herself to the group, and annotations can be made to every item in a CommSy Project Room. Working material can be collected in a simple reference manager and put in context by attaching them to any other item (such as an announced event). A group-editor is available for cooperative writing of HTML documents.

The design principles of CommSy Project Rooms demand (and allow) that the members of a community establish a shared understanding of their working context. For example, they can attach intermediate results to any item in the system, but they must also find ways of making each other aware these results exist. Members have to relate their own communicative purpose to those of others and cannot rely on an automatic facility provided by the software system. In addition, a community must share an understanding about how often the participants use the system and thus read others' items once a week, every second day or more than once a day. If a communication need appears to be too urgent to fit the expected frequency with which other participants use the system, additional communication media, like telephone or e-mail, have to be used.

Three design principles distinguish CommSy Project Rooms from other software products:

- Easy individual use: Enabling individuals to use CommSy Project Rooms easily is a prerequisite for any project member to actively engage in the work without having to overcome technical barriers. We achieve this by implementing only required functionality and a simple, recurring structure across the whole system.

- Responsibility in cooperative usage: CommSy Project Rooms give special emphasis to user communities rather than individuals: CommSy 
Project Rooms are exclusively accessible to members of a certain group and each user's name is recorded with every item s/he creates. Every project room can be customized to help build a group identity by colour selection, by choosing a name for that specific CommSy Project Room, and/ or by selecting a subset of available functions.

- No concept of roles: "Ownership" is the sole access right in CommSy Project Rooms. Only the owner of an item may modify or delete it. Apart from this rule, every member of a CommSy is allowed to do everything and to see everything. There is no distinction between students and teachers. Users can be sure that they get the same presentation as everyone else. In CommSy Project Rooms we promote good social behaviour in our day to day interaction with students stressing self-responsibility and commitment.

CommSy Archive is designed to support teaching and learning individuals over a longer period of time. Users can store and find study materials of any kind. It is possible to select material for special purposes, to leave comments on every item of material or to rearrange it. Every item of material can be labeled as relevant for one or more courses and can be categorized "topic", "kind of material", "author" and "user". Additionally, there is a bibliography for any literature that is not part of CommSy Archive itself, a catalogue of contacts (institutions, organizations and people) and a glossary.

CommSy Archive is characterised by multiple forms of access for users, who have to deal with rapidly changing topics and perspectives. Most of the archive is accessible only to participants of courses using the system. Selected contents are accessible worldwide but, in order to avoid misuse, only a few authorized people have the right to publish files for worldwide access. As a result, the narrow context for tutorial purposes and the wide context for establishing a broad discourse on Social Informatics are effectively separated. The worldwide access is only a part of CommSy Archive situated in a particular learning community, i.e. in a particular knowledge project.

In order to set up CommSy Archive as a part of a knowledge project, it is important to integrate its main archiving functions into virtual and real cooperation settings. CommSy Archive not only supports information retrieval but also serves as a publishing medium. Student authorship is one of the core principles CommSy Archive is designed to support. Students and faculty staff may produce results relevant for a broader discourse and structure material to fulfill different, but specific needs, thus contributing to the context of the future usage of the archive.

Learning communities may take interesting material from CommSy Archive into their CommSy Project Rooms in order to discuss it more intensively and improve it. Thus, material on CommSy Archive potentially 
meets the criterion of "state-of-the-art" because new versions or new papers from CommSy Project Rooms contribute to the archive.

CommSy Campus serves as a portal, providing central access to CommSy Project Rooms and CommSy Archive. User support and other administrative functions are bundled here. Users - students as well as teachers - may register offered courses and extra-curricular activities here, provide information and study materials relevant for their courses and apply for a CommSy Project Room.

A central facility on CommSy Campus is a listing of courses and extracurricular activities in current and earlier semesters, complete with appropriate project rooms and archived study material. Several courses belonging to a common context may be arranged in so-called "minicurricula". CommSy Campus also offers "topics" - a collection and description of relevant subjects which can be attached to courses and archived materials. In addition it offers a notice-board on which announcements can be made.

We consider CommSy as a medium designed to support learning communities. While CommSy Project Rooms focus on learning communities working together on a particular knowledge project, CommSy Archive and CommSy Campus strive to give substantial support over a longer period of time and strengthen connections between different parts and contents of study courses. They therefore support university education in its entirety as a knowledge project.

\section{FUTURE WORK}

Establishing knowledge projects is not only based on the development and use of software systems. In our research and development work, we also consider the following tasks:

- Embedding the software systems pedagogically. Since the use of new media in educational settings is seldom successful without changes in the way we teach we intend to concentrate on ways of embedding CommSy pedagogically in our future work. We will educate faculty staff in the use of the software system.

- Establishing knowledge projects as organizational development. In order to master the technological and organizational aspects of establishing knowledge projects in a university we foster a process of organizational development.

- New didactical paradigms, including devices designed to support learning communities, need careful evaluation. Therefore we are monitoring the processes in our knowledge project from different 
perspectives (evaluation by learners, teachers, consultants, experts).

\section{Acknowledgements}

Christiane Floyd and Horst Oberquelle were extremely generous with their time and inspiration. Thomas Bogner provided very helpful and profound feedback to this piece of work. Special thanks go to the huge number of students who helped us in developing CommSy. Funds for conducting some of the research reported here came from the Zukunftsinvestitionsprogramm of the German Federal Government.

\section{REFERENCES}

CommSy Website (2001) [http://www.commsy.org, 30.10.2001]

Gudjons, H. (1997) Didaktik zum Anfassen: Lehrer/in-Persönlichkeit und lebendiger Unterricht. Klinkhardt, Bad Heilbrunn.

Kling, R. (1999) What is Social Informatics and Why Does it Matter? D-Lib Magazine, 5(1).

Kling, R. and Star, L. (1998) Human Centered Systems in the Perspective of Organizational and Social Informatics. Computers and Society, 28(1).

Pape, B., Bleek, W. -G., Jackewitz, I. and Janneck, M. (2002) Software requirements for project-based learning: CommSy as an exemplary solution. Proceedings of the 35th Hawaii International Conference on System Science.

Rogers, C. (1969) Freedom to learn. Merrill, Columbus, Ohio.

Schultze, U. (1998) Investigating the Contradictions in Knowledge Management. Proceedings of IFIP Working Groups 8.2 and 8.6 Joint Working Conference on Information Systems: Current Issues and Future Changes. 